\title{
Perception of mucormycosis infection among Bangladeshi healthcare workers: an exploratory cross-sectional study in the year following the COVID-19 pandemic
}

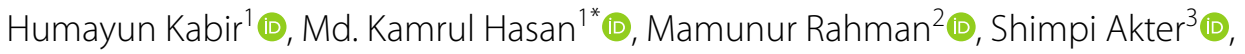 \\ Golam Ishraque Chowdhury ${ }^{4}$ (i), Mohammad Toyabur Rahaman Bhuya ${ }^{5}$ and Dipak Kumar Mitra ${ }^{1}$ (D)
}

\begin{abstract}
Background: Mucormycosis, a severe fungal infection, is an emerging public health concern during the COVID-19 pandemic. This study aimed to investigate the perception of mucormycosis among Bangladeshi healthcare workers.

Results: An exploratory cross-sectional study was carried out among the Bangladeshi healthcare workers from May 25, 2021, to June 5, 2021. The study found 422 responses from the healthcare workers of Bangladesh. Among the respondents, nearly half of them $(45.26 \%)$ were doctors $(n=191)$. This study explored that the healthcare workers' mucormycosis perception scores were significantly associated with their age, gender, profession, monthly income, marital status, job type, and death of friends and family members due to COVID-19.

Conclusions: This study emphasized the healthcare workers' mucormycosis perception along with other associated factors. The findings could help policymakers to mitigate mucormycosis and related infectious diseases emergencies in the post-COVID-19 situation.
\end{abstract}

Keywords: Mucormycosis, Perception, COVID-19, Healthcare workers, Bangladesh

\section{Background}

The COVID-19, Coronavirus disease 2019, also known as SARS-CoV-2, adversely affected Bangladesh as a pandemic with around 1 million cases until July 1, 2021 (Kabir et al. 2021; Worldometer 2021). Recently, the delta variant in Bangladesh has been more transmissible $(60 \%$ more) than the alpha variant, causing steeper infection rates. In the COVID-19 era, another most highlighted topic is mucormycosis, also known as black fungus, which existed to date back to 1855 , emerged recently because the infection rate has elevated due to

\footnotetext{
*Correspondence: kamrul.hasan11@northsouth.edu;

kamrulhasanhridoy205@gmail.com

${ }^{1}$ Department of Public Health, North South University, Plot 15, Block B,

Bashundhara, Dhaka 1229, Bangladesh

Full list of author information is available at the end of the article
}

COVID-19 (Chander 2018; Hasan et al. 2021; Kamruzzaman 2021; Raut and Huy 2021). Mucormycosis, a fungal infection caused by fungi in the order Mucorales, affects people with reduced ability to fight infection and diabetes patients that help the fungi to flourish more (Greene et al. 2008; Skiada et al. 2020). According to Skaida et al., 1.7 people per million were affected by mucormycosis though the infection rate was eighty times higher in India during the COVID-19 (Skiada et al. 2020). Bangladesh detected the first COVID-19 induced mucormycosis case on May 8, 2021, and the death on May 23, 2021, whereas India reported 14,872 cases up to May 28, 2021 (Kamruzzaman 2021; Raut and Huy 2021). The Indian authorities have already stated that mucormycosis should be declared an epidemic due to the higher mortality rate (Staff 2021). However, adequate perception of healthcare 
workers and proper utilization consider practical measures to win in the battle of disease outbreaks (Watkins 2020). Fetansa et al., reported that the level of perception of the healthcare workers regarding any emerging diseases might be varied by different determinants (Fetansa et al. 2021).

World Health Organization (WHO) reported that around 1 out of 9 healthcare workers were affected by the COVID-19 (Linda 2020). Similarly, 11\% of Bangladeshi healthcare workers were affected by 2020 (Islam et al. 2020). This year, mucormycosis has become a severe concern to the COVID-19 survived patients (Anand 2021; Kamruzzaman 2021). Therefore, mucormycosis can be a matter of concern for healthcare workers with a history of COVID-19, as they have to undergo exposure in the clinical field. On the contrary, being geographically attached to India, this became an alarming issue in Bangladesh, where the mortality rate of mucormycosis has risen to $80 \%$ in India (Staff 2021). Overuse of immunosuppressive drugs, corticosteroid therapy for the COVID-19 patients, and having comorbidities were reported to be significant factors for COVID-19 induced mucormycosis emergencies (Ahmadikia et al. 2021; Rahman et al. 2021). Also, unhealthy preventive practices, e.g., reuse of unwashed or one-time mask, could contribute to the sudden upsurge of mucormycosis infection (Sen et al. 2021).

Though mucormycosis has been described, COVID19 associated mucormycosis is mostly rhino orbital cerebral type infection clinically manifested around the nose, eye, and even in the brain (Roden et al. 2005; Sen et al. 2021). Evidence found that immune-compromised patients at post-COVID-19 have been suffered from pulmonary mucormycosis (Alekseyev et al. 2021). However, mucormycosis is not a contagious disease in nature (Ananthaneni et al. 2013). Avoiding the risk factors and administration of antifungal agents along with better management are recognized approaches to manage mucormycosis (Skiada et al. 2018).

As front-line fighters, the Bangladeshi healthcare workers were actively involved in managing the COVID-19 situation. Similarly, they are also willing to fight against the mucormycosis infection related emergencies among COVID-19 survived patients. Thus, proper perception is considered to be the basis of excellence and wisdom in the field of outbreaks (Badran 1995). Inadequate perception of mucormycosis of healthcare workers can lead to inefficient control of the emergencies during this pandemic (Gizaw et al. 2015). In the case of COVID-19, it was found that appropriate perception contributed to managing the pandemic situation with lesser friction (Wake 2020).

Currently, no study has been found that assessed perception of mucormycosis and associated factors among
Bangladeshi healthcare workers during the COVID19 pandemic. Our study investigated the perception of mucormycosis among Bangladeshi healthcare workers with different sociodemographic, work-related, and COVID-19 related factors. The study findings may help the policymakers to respond appropriately and imply the corrective actions by improving the healthcare system of Bangladesh and the situation of the frontline healthcare workers.

\section{Methods}

Study design, settings, and populations

An exploratory cross-sectional study was conducted during the COVID-19 pandemic among the Bangladeshi healthcare workers between May 27, 2021, and June 3, 2021. The inclusion criteria of the respondents include; (a) the registered healthcare workers such as doctors, nurses, and others (technologist, radiologist, and medical assistant), (b) age over 18 years, (c) enrolled at clinical practices, and (d) participated by giving an online consent.

\section{Data collection procedure}

Data were collected by a semi-structured online questionnaire (using a "Google Form) followed by convenience and snowball sampling methods. Due to the COVID-19 pandemic, face-to-face data collection was prohibited. A self-response "Google Form" questionnaire link was distributed on social media platforms or by text messages for data collection. When the participants clicked on the link, they found the study's aim and objective on the front page. The agreed respondents were transferred to the remaining part of the questionnaire. The sociodemographic and workplace-related items were presented on the second and third pages of the questionnaire, respectively. The items related to the perception of mucormycosis were delivered on the fourth page of the questionnaire. Henceforth, a total of 422 completed responses from 468 were included for the final analysis.

\section{Dependent and independent variable measurement}

The dependent variable of this study was the perception of mucormycosis. The independent variables were sociodemographic characteristics (age, gender, residence, profession, monthly income, marital status, having children, and living status), work-related information (job type, working condition, having PPE (personal protective equipment), and direct patient contact), and COVID19 related information (COVID-19 positive status, FNF (friend and family) members' COVID-19 positive status, and FNF COVID-19 members' death). 


\section{Perception of mucormycosis}

To examine the perception of mucormycosis, the authors adopted a six items' measure that is presented in Table 1. The items were responded to a five-point Likert scale; 1 for 'strongly disagree' to 5 for 'strongly agree.' After summing all the items, the total score was ranged from 6 to 30 , whereas a higher score indicated a higher level of positive perception. For content validity, experts reviewed the questionnaire, and, based on their comments, minor modifications were performed. For construct validity, inter items correlations were checked, where the items were correlated significantly. The measures' reliability coefficient, Cronbach $\alpha$, was found 0.79 , indicating an acceptable internal consistency.

\section{Data analysis}

Descriptive statistics were performed to examine the distribution of the studied variables. To find the association between studied variables and mucormycosis perception score, 't-test' and 'one-way ANOVA test' were performed. However, before the t-test, we performed the SD test to assess the equality of variances. Multivariable hierarchical robust regression models were used to predict mucormycosis perception. The $p$ value $<0.05$ was considered statistically significant at a $95 \%$ confident interval. Data were analyzed by using statistical software STATA version 16. The bar charts were illustrated by Microsoft Excel version 16 .

\section{Results}

\section{Descriptive statistics of studied variables}

Descriptive statistics of studied variables are presented in Table 2. A total of 422 healthcare workers participated. Most of them were aged between 24 and 30 years. In terms of gender, $47.63 \%$ was male $(n=201)$. The majority of them $(n=372)$ were from urban areas $(88.15 \%)$. Nearly half of them (45.26\%) were doctors $(n=191)$. Maximum of the participants $(54.27 \%)$ were married $(n=229)$. The participants' having children $(n=106)$ was $25.12 \%$ and living alone $(n=89)$ was $21.09 \%$. The majority of them $(n=277)$ did the private job $(65.64 \%)$ and $31.52 \%$ worked in COVID-19 conditions $(n=133)$. Most of them $(n=275)$ had a lack of PPE $(65.17 \%)$, whereas having direct patient contact $(n=316)$ was $74.88 \%$. Almost twothirds $(n=318)$ of them got COVID-19 infection (76\%). On the other hand, $22.09 \%$ of the participants' FNF got COVID-19 infection $(n=381)$ and died of COVID-19 infection $(n=93)$ was $22.09 \%$.

\section{Association between studied variables and mucormycosis perception}

The association between the studied variables and the mucormycosis perception is presented in Table 2. Alongside the distribution of the responses of mucormycosis perception measure is illustrated in Fig. 1. Age was found significantly associated with mucormycosis perception $(F=5.34, p=0.001)$. The mucormycosis perception score was significantly higher among the female healthcare workers than males $(t=2.53, p=0.012)$. In terms of profession, this study observed a significant difference in mucormycosis perception $(F=14.47, p<0.001)$. The monthly income was significantly associated with the mucormycosis perception $(F=14.47, p<0.001)$. The married healthcare workers had significantly higher perception scores than unmarried $(t=-3.12, p=0.002)$. The government jobholders had significantly higher perception scores than the private $(t=2.41, p=0.016)$. The mucormycosis perception scores were significantly higher among the healthcare workers whose FNF died due to COVID-19 $(t=-1.98, p=0.049)$.

\section{Predictive models of mucormycosis perception}

Multivariable hierarchical robust regression models are presented in Table 3. In Block 1, the demographic variable explained an $11 \%$ variance of the perception score. Adding work-related variables in Block 2 explained 2\% of the variance. After adding COVID-19 related variable in the final model (Block 3), a total explanatory variance was fixed to $14 \%$.

Table 1 The items of mucormycosis perception measure

\begin{tabular}{ll}
\hline Denotes & Items \\
\hline P-1 & Mucormycosis is known as black fungus. \\
P-2 & The COVID-19 increases the risk of mucormycosis infection. \\
P-3 & Steroids treatment attributed risk of mucormycosis infection. \\
P-4 & Immunosuppressive overuse could be a reason for mucormycosis infec- \\
P-5 & tion. \\
P-6 & Having comorbidities are the risk factor of mucormycosis infection. \\
\hline
\end{tabular}


Table 2 Association between studied variables and mucormycosis perception

\begin{tabular}{llll}
\hline Variables & $n(\%)$ & Perception & \\
\cline { 3 - 4 } & Mean and SD & $\begin{array}{l}F / t \text { test } \\
\text { value }\end{array}$ & $p$-value \\
& &
\end{tabular}

Sociodemographic variables

Age (in year)

$<24$

24-27

$18(4.27)$

$21.89 \pm 4.90$

5.34

28-30

$168(39.81) \quad 24.15 \pm 4.77$

$>31$

$146(34.60) \quad 24.86 \pm 4.59$

Gender

Male

Female

$90(21.33) \quad 25.89 \pm 3.65$

Residence

Rural

Urban

Profession

Doctor

Nurse

$24.09 \pm 4.77$

$221(52.37) \quad 25.20 \pm 4.32$

Others

$50(11.85)$

$372(88.15)$

$25.24 \pm 4.22$

$24.59 \pm 4.61$

$191(45.26) \quad 25.88 \pm 3.89$

$161(38.15)$

$24.0 \pm 4.91$

Income (monthly)

$<20,000$ BDT

20000

30000 BDT

$>30000$ BDT

$70(16.59)$

$22.91 \pm 4.63$

Marital status

Unmarried

Married

Having children

Yes

No

$89(21.09) \quad 22.67 \pm 4.88$

$163(38.63)$

$24.85 \pm 4.59$

$170(40.28) \quad 25.55 \pm 4.07$

Living status

Alone

$193(45.73) \quad 23.92 \pm 4.77$

$-3.12$

$229(54.27) \quad 25.30 \pm 4.30$

With others

$106(25.12) \quad 25.20 \pm 4.07$

$316(74.88)$

$24.49 \pm 4.72$

$-1.48$

0.140

Work-related variables

Job type

$\begin{array}{lllll}\text { Government } & 145(34.36) & 25.41 \pm 4.31 & 2.41 & \mathbf{0 . 0 1 6} \\ \text { Private } & 277(65.64) & 24.29 \pm 4.66 & & \\ \text { Working condi- } \\ \text { tion }\end{array}$

Table 2 (continued)

\begin{tabular}{|c|c|c|c|c|}
\hline \multirow[t]{2}{*}{ Variables } & \multirow[t]{2}{*}{$n(\%)$} & \multicolumn{3}{|l|}{ Perception } \\
\hline & & Mean and SD & $\begin{array}{l}F / t \text { test } \\
\text { value }\end{array}$ & $p$-value \\
\hline Yes & $118(27.96)$ & $25.11 \pm 4.78$ & -1.23 & 0.218 \\
\hline No & 304 (72.04) & $24.5 \pm 4.48$ & & \\
\hline \multicolumn{5}{|c|}{$\begin{array}{l}\text { FNF COVID-19 } \\
\text { positive }\end{array}$} \\
\hline Yes & 381 (75.53) & $24.58 \pm 4.70$ & 0.42 & 0.208 \\
\hline No & $103(24.47)$ & $25.00 \pm 4.13$ & & \\
\hline \multicolumn{5}{|c|}{$\begin{array}{l}\text { FNF COVID-19 } \\
\text { death }\end{array}$} \\
\hline Yes & 93 (22.09) & $25.51 \pm 4.48$ & -1.98 & 0.049 \\
\hline No & $328(77.91)$ & $24.45 \pm 4.57$ & & \\
\hline
\end{tabular}

\section{Discussion}

This study found that the overall perceptions level of mucormycosis was significantly associated with the healthcare workers' age, gender, marital status, profession, monthly income, marital status, job type, and FNF death due to COVID-19. Similarly, numerous studies were conducted previously to assess the healthcare workers' perception level in different communicable diseases emergencies (Gizaw et al. 2015; Ejeh et al. 2020; Wake 2020; Dil et al. 2020; Almohammed et al. 2021; Fetansa et al. 2021; and Roupa et al. 2021).

Age was found significantly associated with mucormycosis perception. The highest mean of perception score was observed in the higher age group. Similarly, Roupa et al., found that the perception of COVID-19 was significantly higher among the older age group than the younger age group (Roupa et al. 2021). However, for healthcare workers, aging may help gaining experiences and more perception regarding any diseases (Mahmud et al. 2020).

In this study, gender was significantly associated with the perception score of mucormycosis. Female healthcare workers were perceptually more sound. A previous survey of COVID-19 indicated that the perception of female healthcare workers was more adequate than that of male healthcare workers (Almohammed et al. 2021). Moreover, Nebhinani and Saini revealed that female healthcare workers more strongly perceived NCD (non-communicable disease) related information (Nebhinani and Saini 2020).

The profession was found to be significantly associated with the perception of mucormycosis. We found that the doctors had the highest perception score compared to other healthcare workers. Similarly, Dil et al., 
P-1

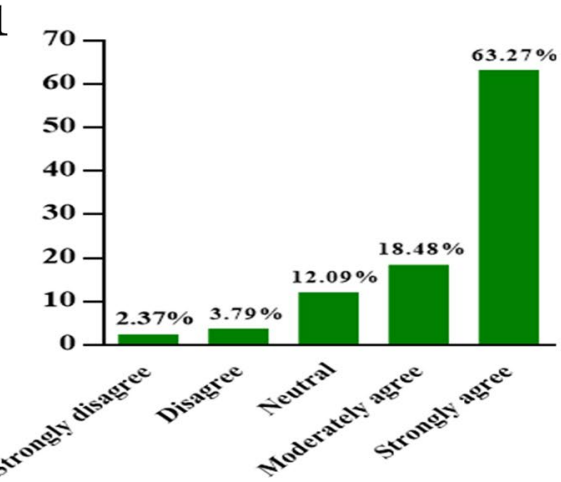

P-3

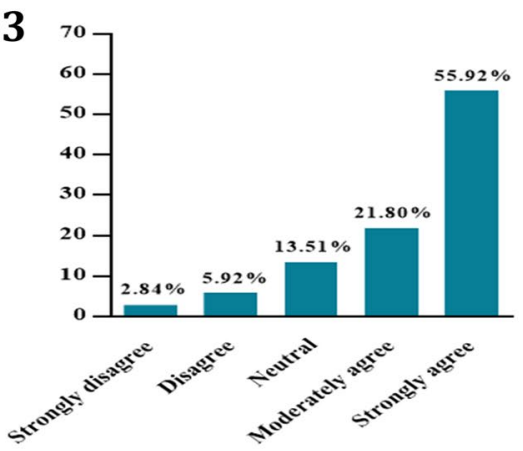

P-5

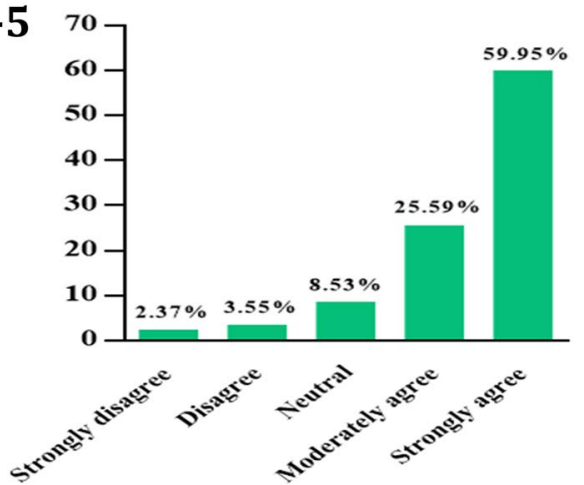

P-2

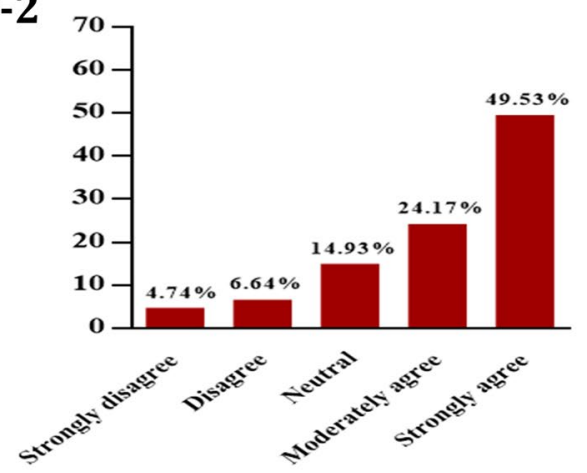

P-4

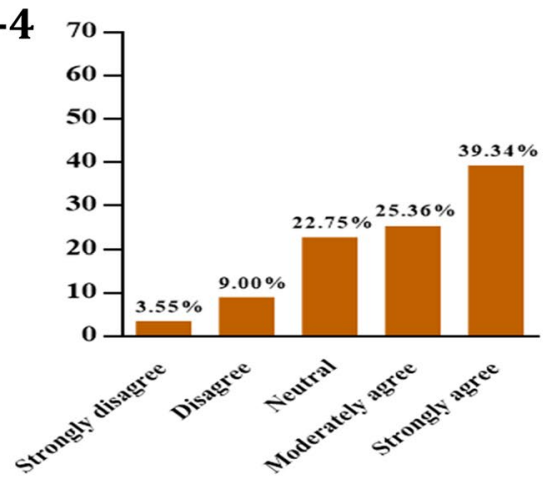

P-6

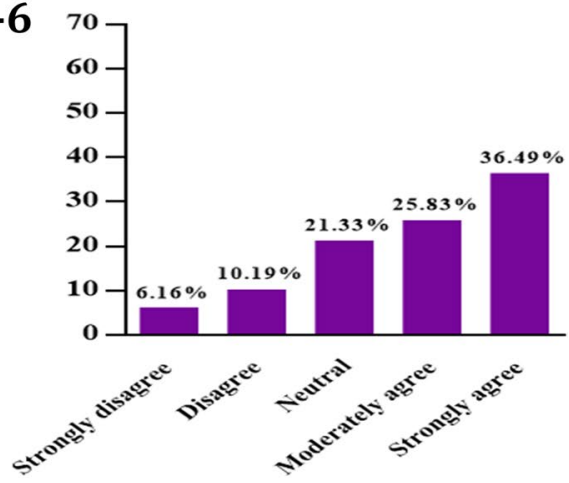

Fig. 1 The distributions of six items' (denoted by P-1 to P-6) mucormycosis perception measure

regarding COVID-19 found that doctors had higher perception scores than other healthcare workers (Dil et al. 2020). Kanu et al., also reported that the physicians were more perceptional on the coronavirus disease than the community healthcare workers and medical technicians (Kanu et al. 2021).

This study found that married healthcare workers were more perceptional compared to unmarried healthcare workers. Studies on the coronavirus also supported our findings that married healthcare workers scored more on the coronavirus perception scale (Ejeh et al. 2020; Tsiga et al. 2021). However, Palner and Mittelmark stated that married people were mentally more vital for gaining proper health related perception (Palner and Mittelmark 2009).

The healthcare workers with government jobs were more perceptional than the private job holder healthcare workers. Similarly, Basu et al., described that the public healthcare workers were more efficient in disease perception (Basu et al. 2012). In this study, the FNF COVID-19 death was significantly associated with the perception of mucormycosis. The death of friends or family may cause anxiety in one individual life. Fitri et al., reported that social curiosity may propel acquiring more perception that helps eradicate death anxiety (Fitri et al. 2020). 
Table 3 Multivariable hierarchical robust regression models to predict mucormycosis perception

\begin{tabular}{|c|c|c|c|}
\hline Variables & Block $1(\beta)$ & Block $2(\beta)$ & Block $3(\beta)$ \\
\hline Age (in year) & 0.25 & 0.15 & 0.14 \\
\hline Gender & $-1.18^{* *}$ & $-1.08^{*}$ & $-1.09^{*}$ \\
\hline Residence & -1.02 & -1.10 & -0.98 \\
\hline Profession & $-0.97^{* *}$ & $-1.14^{* *}$ & $-1.23^{* *}$ \\
\hline Income (monthly) & $0.82^{*}$ & 0.69 & $0.85^{*}$ \\
\hline Marital status & 0.98 & 1.01 & 0.99 \\
\hline Having children & -0.62 & -0.61 & -0.59 \\
\hline Living status & -0.63 & -0.58 & -0.55 \\
\hline Tob type & & -0.72 & -0.84 \\
\hline Working conditions & & -0.09 & -0.14 \\
\hline Having PPE & & 0.72 & 0.63 \\
\hline Direct patient contact & & -0.18 & -0.23 \\
\hline COVID-19 positive & & & 0.27 \\
\hline FNF COVID-19 positive & & & $-1.49^{* *}$ \\
\hline FNF COVID-19 death & & & $0.03^{*}$ \\
\hline$F$ & 6.99 & 5.29 & 4.55 \\
\hline$R^{2}$ & 0.11 & 0.12 & 0.14 \\
\hline
\end{tabular}

FNF, friend and family

${ }^{* * *} P<0.001,{ }^{* *} P<0.01,{ }^{*} P<0.05$

In general, assessment of healthcare workers' mucormycosis perception in terms of different determinants may strengthen the healthcare system of Bangladesh. Therefore, to identify the factors behind the different levels of perception regarding any emerging infectious disease and taking corrective actions accordingly is essential.

\section{Strengths and limitations}

To best of our knowledge, this is the first study that attempted to explore the mucormycosis perception among healthcare workers in Bangladesh. A better understanding of this issue can come in handy in implementing prevention measures more effectively with the emergence of mucormycosis like fungal infection.

However, there were several limitations of this study. Due to COVID-19, it was not possible to execute face-toface interviews of participants. Thus, selection bias might be occurred owing to unintentional ruling out the healthcare workers who has no internet access. Besides, since this is a cross-sectional study, it was not possible to draw any cause-effect relationship. In addition, the usage of self-reported questionnaires might pose reporting bias.

\section{Conclusions}

Mucormycosis is not a new infectious disease, but the emergence of mucormycosis in the COVID-19 global crisis is undoubtedly an alarming issue. In this study, the authors tried to take a snapshot of the mucormycosis perception of Bangladeshi healthcare workers. Mucormycosis perception significantly varied with respondents' age, gender, profession, monthy income, marital status, job type, COVID-19 positive, and FNF COVID-19 death. As healthcare workers are the frontline fighters worldwide in the COVID-19 pandemic, a clear perception of mucormycosis is a sine qua non.

\section{Recommendations}

The findings could help policymakers to mitigate mucormycosis and other infectious diseases emergencies in the post-COVID-19 period and pandemic-like condition(s). This study's findings can help tailoring the preventive strategies to mitigate the health emergency in Bangladesh and generalize for other developing countries. Therefore, to overcome the health catastrophe, the policymakers can take the appropriate initiative to disseminate information about mucormycosis through training courses or online webinars with attachments to healthcare workers and emphasize the role of mass media.

\section{Abbreviations \\ FNF: Friend and family; COVID-19: Corona Virus-19.}

\section{Acknowledgements}

We would like to accolade all the research assistants of the project who assisted in data collection.

\section{Authors' contributions}

Conceptualization: $\mathrm{MH}, \mathrm{HK}$; methodology: $\mathrm{HK}, \mathrm{MH}$; validation and scrutinization: DM, HK, MH; investigation: $\mathrm{MH}, \mathrm{HK}$; writing —original draft preparation: HK, MH, MR, SA, GC, MT; review and editing: DM, MH, HK; supervision: DM. HK and $\mathrm{MH}$ contributed equally to this research. All authors have read and agreed to the current version of the manuscript

\section{Funding}

No funding from any public, private or non-profit research agency was received for this study.

\section{Availability of data materials}

Dataset used in this study will be available as per request (mailing to the corresponding author).

\section{Declarations}

\section{Ethics approval and consent to participate}

The Institutional Review Board (IRB) of North South University, Bangladesh, approved the current study. The review ID is 2021/OR-NSU/IRB/0603. The study purpose was explained on the first page of the survey, and the respondents were asked on the first question whether they were willing to participate in the survey. Those selected 'yes' were considered to participate in the study.

\section{Consent for publication}

Not applicable.

\section{Competing interest}

The authors report no competing interest. The authors alone are responsible for the content and writing of this article. 


\section{Author details}

1 Department of Public Health, North South University, Plot 15, Block B, Bashundhara, Dhaka 1229, Bangladesh. ${ }^{2}$ Department of Pharmacy, East West University, Dhaka 1212, Bangladesh. ${ }^{3}$ Bangladesh University of Professionals, Mirpur Cantonment, Dhaka 1216, Bangladesh. ${ }^{4}$ Ministry of Health and Family Welfare, Dhaka, Bangladesh. ${ }^{5}$ Institute of Social Welfare and Research, University of Dhaka, Dhaka 1000, Bangladesh.

Received: 19 October 2021 Accepted: 31 December 2021

Published online: 15 January 2022

\section{References}

Ahmadikia K, Hashemi SJ, Khodavaisy S et al (2021) The double-edged sword of systemic corticosteroid therapy in viral pneumonia: a case report and comparative review of influenza-associated mucormycosis versus COVID19 associated mucormycosis. Mycoses 64:798-808. https://doi.org/10. 1111/MYC.13256

Alekseyev K, Didenko L, Chaudhry B (2021) Rhinocerebral mucormycosis and COVID-19 pneumonia. J Med Cases 12:85-89. https://doi.org/10.14740/ jmc3637

Almohammed OA, Aldwihi LA, Alragas AM et al (2021) Knowledge, attitude, and practices associated with COVID-19 among healthcare workers in hospitals: a cross-sectional study in Saudi Arabia. Front Public Heal 9:1007. https://doi.org/10.3389/FPUBH.2021.643053

Anand SBS (2021) Rare 'Black Fungus,' mucormycosis, infects thousands of Covid-19 survivors in India. In: WSJ. https://www.wsj.com/articles/rareblack-fungus-mucormycosis-infects-thousands-of-covid-19-survivors-inindia-11621614697. Accessed 24 Jul 2021

Ananthaneni AR, Undavalli SB, Velagapudi RP, Guduru VS (2013) Mucormycosis: an atrocious mate of patients with diabetes. Case Rep. https://doi.org/10. 1136/BCR-2013-009600

Badran IG (1995) Knowledge, attitude and practice the three pillars of excellence and wisdom: a place in the medical profession. EMHJ East Mediterr Heal J 1:8-16. https://apps.who.int/iris/handle/10665/116905

Basu S, Andrews J, Kishore S et al (2012) Comparative performance of private and public healthcare systems in low- and middle-income countries: a systematic review. PLoS Med 9:19. https://doi.org/10.1371/JOURNAL. PMED.1001244

Chander J (2018) Textbook of medical mycology. Jagdish Chander. Google Books. In: Heal. Sci. Publ.

Dil L, Rano P, Avinash S (2020) Healthcare workers' knowledge, attitude and practices during the COVID-19 pandemic response in a tertiary care hospital of Nepal. PLoS ONE 15:e0242126. https://doi.org/10.1371/journ al.pone.0242126

Ejeh FE, Saidu AS, Owoicho S et al (2020) Knowledge, attitude, and practice among healthcare workers towards COVID-19 outbreak in Nigeria. Heliyon 6:e05557. https://doi.org/10.1016/j.heliyon.2020.e05557

Fetansa G, Etana B, Tolossa T et al (2021) Knowledge, attitude, and practice of health professionals in Ethiopia toward COVID-19 prevention at early phase. SAGE Open Med 9:205031212110122. https://doi.org/10.1177/ 20503121211012220

Fitri RA, Asih SR, Takwin B (2020) Social curiosity as a way to overcome death anxiety: perspective of terror management theory. Heliyon 6:e03556. https://doi.org/10.1016/j.heliyon.2020.e03556

Gizaw GD, Alemu ZA, Kibret KT (2015) Assessment of knowledge and practice of health workers towards tuberculosis infection control and associated factors in public health facilities of Addis Ababa, Ethiopia: a cross-sectional study. Arch Public Heal 73:1-9. https://doi.org/10.1186/ S13690-015-0062-3

Greene J, Pak J, Tucci V et al (2008) Mucormycosis in immunochallenged patients. J Emergencies Trauma Shock 1:106. https://doi.org/10.4103/ 0974-2700.42203

Hasan MK, Kabir H, Rahman M et al (2021) Association between insomnia and mucormycosis fear among the Bangladeshi health care workers: a cross sectional study. J Affect Disord Reports 6:100262. https://doi.org/10 1016/j.jadr.2021.100262

Islam S, Islam R, Mannan F et al (2020) COVID-19 pandemic: an analysis of the healthcare, social and economic challenges in Bangladesh. Prog Disaster Sci 8:100135. https://doi.org/10.1016/J.PDISAS.2020.100135
Kabir H, Nasrullah SM, Hasan MK et al (2021) Perceived e-learning stress as an independent predictor of e-learning readiness: results from a nationwide survey in Bangladesh. PLoS ONE 16:e0259281. https://doi.org/10.1371/ journal.pone.025928

Kamruzzaman M (2021) Bangladesh detects 2 cases of black fungus in capital. In: Anadolu Agency. https://www.aa.com.tr/en/asia-pacific/bangladeshdetects-2-cases-of-black-fungus-in-capital/2253078. Accessed 24 Jul 2021

Kanu S, James PB, Bah AJ et al (2021) Healthcare workers' knowledge, attitude, practice and perceived health facility preparedness regarding covid-19 in Sierra Leone. J Multidiscip Healthc 14:67-80. https://doi.org/10.2147/ JMDH.S287156

Linda L (2020) How to protect health workers now: WHO COVID-19 briefing. In:World Econ. Forum. https://www.weforum.org/agenda/2020/04/10april-who-briefing-health-workers-covid-19-ppe-training/

Mahmud MS, Begum MK, Akhter J (2020) Knowledge and attitude of senior staff nurses regarding geriatrics health care in a tertiary public hospital. Asian J Med Biol Res 6:431-439. https://doi.org/10.3329/ajmbr.v6i3.49791

Mucormycosis. In: Nord (National Organ. Rare Disord. https://rarediseases.org/ rare-diseases/Mucormycosis/. Accessed 24 Jul 2021

Nebhinani M, Saini SK (2020) Knowledge, skills of female health workers regarding selected non communicable diseases risk reduction and client satisfaction: a pilot study from western part of Rajasthan. India. Int J Res Med Sci 8:2802. https://doi.org/10.18203/2320-6012.ijrms20203089

Palner J, Mittelmark MB (2009) Differences between married and unmarried men and women in the relationship between perceived physical health and perceived mental health. Nor Epidemiol 12:55-61. https://doi.org/10. 5324/nje.v12i1.507

Rahman F, Islam R, Bhuiyan M (2021) Mucormycosis or black fungus infection is a new scare in South Asian countries during the COVID-19 pandemic: associated risk factors and preventive measures. J Med Virol 93:64476448. https://doi.org/10.1002/jmv.27207

Raut A, Huy N (2021) Rising incidence of mucormycosis in patients with COVID-19: another challenge for India amidst the second wave? Lancet Respir Med 9:e77. https://doi.org/10.1016/S2213-2600(21)00265-4

Roden MM, Zaoutis TE, Buchanan WL et al (2005) Epidemiology and outcome of zygomycosis: a review of 929 reported cases. Clin Infect Dis 41:643653. https://doi.org/10.1086/432579

Roupa Z, Polychronis G, Latzourakis E et al (2021) Assessment of knowledge and perceptions of health workers regarding COVID-19: a cross-sectional study from Cyprus. J Community Health 46:251-258. https://doi.org/10. 1007/s10900-020-00949-y

Sen M, Honavar SG, Sharma N, Sachdev MS (2021) COVID-19 and eye: a review of ophthalmic manifestations of COVID-19. Indian J Ophthalmol 69:488-509. https://doi.org/10.4103/ijo.IJO_297_21

Skiada A, Lass-Floerl C, Klimko N et al (2018) Challenges in the diagnosis and treatment of mucormycosis. Med Mycol 56:93-101. https://doi.org/10. 1093/mmy/myx101

Skiada A, Pavleas I, Drogari-Apiranthitou M (2020) Epidemiology and diagnosis of mucormycosis: an update. J Fungi 6:265. https://doi.org/10.3390/ JOF6040265

Staff SR (2021) India declares 'Black Fungus" epidemic as infections rise among Covid-19 patients.'In: Forbes. https://www.forbes.com/sites/siladityaray/ 2021/05/20/india-declares-black-fungus-epidemic-as-infections-riseamong-covid-19-patients/?sh=3638141210af

Tsiga FI, Amole TG, Musa BM et al (2021) COVID 19: evaluating the knowledge, attitude and preventive practices of healthcare workers in Northern Nigeria. Int J MCH AIDS 10:88-97. https://doi.org/10.21106/ijma.418

Wake AD (2020) Knowledge, attitude, practice, and associated factors regarding the novel coronavirus disease 2019 (COVID-19) pandemic. Infect Drug Resist 13:3817-3832. https://doi.org/10.2147/IDR.S275689

Watkins J (2020) Preventing a covid-19 pandemic. BMJ 368. https://doi.org/10. 1136/BMJ.M810

Worldometer (2021) Bangladesh COVID: 1,153,344 cases and 19,046 deaths. https://www.worldometers.info/coronavirus/country/bangladesh/. Accessed 25 Jul 2021

\section{Publisher's Note}

Springer Nature remains neutral with regard to jurisdictional claims in published maps and institutional affiliations. 